\title{
Reflections on Advanced Practice of Nurse Administered ECT as a Treatment Resource during the COVID-19 Pandemic
}

\author{
Lorraine Bastick, Tracey Shrimpton
}

The Treatment Centre, Northampton Healthcare NHS foundation Trust, Berrywood Hospital, Northampton, UK Email: Lorraine.bastick@nhft.nhs.uk, Tracey.shrimpton@nhft.nhs.uk

How to cite this paper: Bastick, L. and Shrimpton, T. (2021) Reflections on Advanced Practice of Nurse Administered ECT as a Treatment Resource during the COVID19 Pandemic. Open Journal of Nursing, 11, 909-919.

https://doi.org/10.4236/ojn.2021.1111074

Received: June 2, 2021

Accepted: November 7, 2021

Published: November 10, 2021

Copyright $\odot 2021$ by author(s) and Scientific Research Publishing Inc. This work is licensed under the Creative Commons Attribution International License (CC BY 4.0).

http://creativecommons.org/licenses/by/4.0/

\begin{abstract}
Background: With Electro-Convulsive Therapy (ECT) during the COVID-19 pandemic at risk, nurse administered ECT has become a treatment resource. This study aims to investigate the experiences of two senior ECT nurses practicing Nurse Administered ECT. Method: Qualitative reflective interviews were conducted; the data was then analyzed and presented as corresponding themes for inductive data driven analysis. Each nurse was asked to recall positive and negative events from their practice and describe how their experiences have enabled them to cope. Results: Theme 1: The nurse's competence is positively influenced by feedback, leading to enhancement of personal and professional development. Theme 2: During the COVID-19 pandemic, nurses report an increased sense of teamwork among staff, and feel that "we are all in this together". Theme 3: The nurse's ability to manage difficult situations is dependent on their education, individual knowledge, and experience. Limitations: The authors had the role of both researcher and participant. Conclusion: This study found that both nurses valued feedback above any medical and technical skills they had acquired. This strengthened their advanced practice enabling them to handle difficult situations with confidence. The COVID 19 pandemic has led to advanced nursing practice replacing the junior doctor role within ECT treatment. The nurses felt this had been recognised by their organisation and has led to an increased pride in their work. This paper would suggest that Nurse administered ECT is a valuable resource and that, future nurses could be at the forefront of delivering ECT services.
\end{abstract}

\section{Keywords}

ECT, Nurse Administered ECT 


\section{Introduction}

The COVID-19 pandemic has required a massive transformation in UK healthcare services that has triggered a sudden shift in the nursing role. As an increased demand for COVID related care has put pressure on an already deficient workforce creating additional pressures on health provision within the NHS. As the pandemic reached its peak, a lack of anaesthetists, operating departmental practitioners (ODP) and recovery nurses arose, and hospitals began closing down ECT services. People with serious mental health issues are at high risk, and timely access to ECT at this time is vital. A treatment is often a last resort for the most severely psychiatrically unwell [1] [2] [3] [4] [5].

The advanced practice psychiatric nurse has evolved as a resource for healthcare [6]. Advanced practice is a field in which nurses work as an expert within their defined roles. They provide specialist care, complex decision making skills with advanced clinical competencies improving care for their patients, whilst still providing the basis care a novice nurse would provide [7]. It is defined in its terms from the Advanced Practice Nurse training program. Within the field of ECT, this advanced practice may have a key role to play in preserving and expanding ECT treatment when the existing facilities have become disturbingly sparse [8]. ECT is a specialised treatment that requires a close working relationship between the ECT consultant psychiatrist and the ECT nursing team. Nurse administered ECT has evolved over the last 10 years and a bespoke training package for nurses to administer ECT in place of doctors [9] was approved by ECTAS in 2016. This training was completed by the two authors (senior nurses). The authors found a lack of evidence comparing quality of care and outcomes between doctor administered ECT and nurse administered ECT practice; however, further research into advanced nursing practice suggests that psychiatrists time would be more efficiently spent in the management of patients with complex needs, whereas it has been found the needs of less complex patients can be met by advanced practice nurses [6] [8].

The two authors (senior nurses) completed the Leicester University VLE Core Trainees assessment. Both nurses completed the course successfully and passed onto the Practical assessment outlined by the ECTAS Core Trainee Competencies. This was completed over a period of 4 weeks, which resulted in the nurses gaining confidence and competence. The training took 3 months to complete alongside the normal running of the unit duties. Over this period, supervision from the consultant was given after each ECT treatment session initially and subsequently every month or as and when required.

Within the time, both nurses practiced Nurse administered ECT. There has only been one incident noted: a patient experienced a prolonged seizure of which the team struggled to reverse with Midazolam. This incident was investigated, and no causation could be found. The patient was not harmed and recovered fully to be discharged from hospital.

The study took place at the Treatment Centre, Berrywood Hospital, Nor- 
thampton, which is a stand-alone unit within a mental health hospital. They have been accredited with merit by the ECTAS accreditation standards. During COVID-19, despite great difficulties, they were able to maintain a reduced ECT service of a once weekly session treating 3 patients (35\% capacity) for 3 months post COVID lockdown. Fortunately, from early July 2020, twice weekly ECT regime was resumed, although still at the reduced capacity.

From the start of the outbreak, input from junior doctors was very limited as they were often needed elsewhere within the hospital. Junior doctors time had already been impacted by the European working time directive which reduced their assigned time for training, advanced nursing practice bridged the gap created. They were working under considerable increase in clinical workload; some were working in other hospital sites, unable to travel to the Berrywood ECT site due to COVID infection control restrictions. It was this issue that led to the service developing into a nurse administered only service.

This study aims to investigate how the practice of Nurse Administered ECT has become a treatment resource during the COVID-19 pandemic by means of collating "red thread" themes from the reflective accounts of two senior ECT nurses that practice nurse administered ECT within a mental health hospital in the UK.

\section{Data Collection}

The NMC define being a reflective practitioner as:

Reflection is the thought process where individuals consider their experiences to gain insights about their whole practice. Reflection supports individuals to continually improve the way they work or the quality of care they give to people. It is a familiar, continuous, and routine part of the work of health and care professionals [10].

Data was collected from the reflective accounts of the two practitioners using a qualitative approach. Resources for candidates to select were limited to staff trained in administering ECT of which only two staff were suitable. The questions were kept short and specific to allow autonomous feedback and to focus on the candidate's personal thoughts and feelings to exclude any influence or bias from direct questioning. The collected data was then analyzed and presented as themes, corresponding to the interview questions as below:

(Q1) Describe a positive event from your nurse administered ECT practice that occurred during the COVID pandemic and how you're experience/competence/ training has enabled you to manage the event.

(Q2) Describe a negative event from your nurse administered ECT practice that occurred during the COVID pandemic and how you're experience/competence/ training has enabled you to manage the event.

The interviewees were both female, age 52 and age 54, combined experience in nursing 23 years and ECT experience 10 years. The reflective accounts took several hours to complete over a period of two days. 


\section{Analysis}

Together the reflections were read through and reviewed by both authors. It was identified that the analysis of content needed a slightly different approach to the traditional Critical Incident Technique qualitative analysis [11]. It was decided a qualitative content analysis method approach was required [12] [13] and a more inductive data-or text driven analysis which is characterized by identification of patterns within the content of the reflections. This involves the researcher identifying similarities and differences in the data, which are described in categories and or themes at various levels of abstraction and interpretation. Morse [14] described a theme as a meaningful essence that runs through the data, sometimes in the background and sometimes in the foreground.

\section{Results}

\begin{tabular}{|c|c|}
\hline Theme one & Positive feedback enhances personal and professional development \\
\hline Theme two & $\begin{array}{l}\text { During the COVID pandemic nurses report an increased sense of } \\
\text { teamwork among staff, with team members helping one another. }\end{array}$ \\
\hline Theme three & $\begin{array}{l}\text { The nurse's ability to manage psychologically trying situations and } \\
\text { ethical dilemmas are dependent on education, individual knowledge } \\
\text { and experience }\end{array}$ \\
\hline
\end{tabular}

\section{Results for the positive reflective accounts.}

Theme 1: The nurse's competence, in this case nurse administered ECT, is positively influenced by feedback from patients and colleagues leading to enhancement of personal as well as professional development.

"For me the positives have been when you explain to the patient that you are doing the treatment and they turn and say they would rather have you than a doctor as they know me".

" $A$ patient holding my hand and telling me they are glad I am administering her ECT at a time when COVID is at its height and she felt nervous and scared just coming to the treatment centre"

"The team you work with are happy that you have completed the training and give you encouragement to do the "doctors role". The anesthetist saying $1 \mathrm{~m}$ going a great job. How the team are always given praise from me and everything has run smoothly and on time".

"Being asked to contribute to the training of our new Consultant"

"Having my first supervision with our Consultant and he tells us both we are doing well, and we did not discuss any problems just how we go forward".

Theme 2: During the COVID pandemic nurses report an increased sense of teamwork among staff, with team members helping one another and feeling that "we are all in this together".

"At the start of the pandemic knowing there was a possibility that the centre would close due to the anesthetist being needed at NGH. This put immense pressure on us to keep the centre open even though it was for only one day a 
week for 6 months and treating on 3 patients at a time".

"Being stopped in the hospital corridor by a CT doctor during the height of the pandemic telling me he really appreciates our help by releasing him from ECT duties when doctors were experiencing significant increase in work pressure. I felt proud to be helping out the doctors, that my skills and knowledge were being put to good use; I felt I had been acknowledged that our advanced nursing practice had made a difference. I felt we were out there doing our thing holding every-thing together to bring a service to some of the most psychiatrically unwell patients".

"Our NA training had been relaxed and unrushed until the outbreak now we had to stand up to the challenge of administering on our own in the room with the consultant outside due to numbers in the room being kept to a minimum as per AGP guidance. The whole team seemed to come together knowing we need to support each other, that feeling of unity and interconnection; I feel that was reassuring to the patient and that she understood these were difficult times. I became a nurse to fulfil that need to help people and it felt good to do this at a time when the world was in chaos".

\section{Results for the Negative Reflective Accounts}

Theme 3: The nurse's ability to manage psychologically trying situations and ethical dilemmas are dependent on education, individual knowledge and experience.

"When a patient is referred, and you and the team feel ECT is not appropriate. Having the confidence to challenge and explain the criteria for this invasive procedure.

"Difficulty getting the impedance before treatment, I felt the pressure of the fact I may never being able to get the impedance under $2000 \mathrm{ohms}$ and the patient would have been under an anesthetic needlessly without treatment. Whilst training I was supported throughout, but there comes a time when you have to do this on your own and this was fast-tracked due to the pandemic. I had built up quite a lot of experience administering ECT, but I feel the procedure had not yet become second nature and that this could affect my confidence".

"When a patient arrives for the anesthetic review/ treatment and the referring team still haven't completed all the require test/ paperwork and you must explain that you cannot treat. You feel for the patient. However, you are doing your job and keeping the patient safe. I sometime feel those doctors, nurses and other colleagues, we are asking for tests and questions that are not necessary. Making them understand that this is required for the anesthetic review and it s the anesthetist decision to treat once we have all this information"

\section{Analysis}

The qualitative analysis resulted in the construction of three themes for discussion. 
Theme 1: A nurse's competence, in this case nurse administered ECT, is positively influenced by feedback from patients and colleagues leading to enhancement of personal and professional development.

Reflection and self-evaluation from all forms of feedback are shown to enhance personal, as well as professional development [15]. The ability to effectively communicate within the treating team not only affect patient outcome but also influences a nurse's self-evaluation and the development of competence and effectiveness. However, how well these are put into practice is influenced by a how well an organisation functions and the professional competence and attitude of their colleagues [15] [16]. Nurses are found to place communication, reflection and behavior skills above the traditional highly valued medical and technical skills endorsed by todays education system [17]. This was supported by Piil et al. [18] they investigated among other things advanced nurses perceptions of their work and found that the nurses did not view themselves a replacement for doctors but that working autonomously they grew in self-confidence and professional identity.

Regular feedback from ECT patients is difficult as they are some of the most psychiatrically unwell patients, both nurses displayed a close professional relationship with their patients, valuing their thoughts and expressed feelings. It has been found that advanced nurse practice produces a high patient satisfaction rate, mainly due to the nurse devoting more time with each patient [6] [19] [20], most studies have not found any negative impact on patient outcomes following the transfer of certain tasks from doctors to nurses [6] [16] [21] [22]. There is limited research on the efficacy of nurse administered ECT [10] [20] and there are no studies about patient feedback, an area that suggests further examination.

Advanced nurse practitioners within ECT have a vital role to play in preserving and developing future ECT services. This is reinforced by policy Modernising Nursing Careers [23] encourages nurses to move towards a more expert autonomous position using evidenced based care and critical analytical practice. This paper would suggest that Nurse administered ECT is still in its infancy; future direction is yet to be discovered; advanced practising ECT nurse could be able to accept patient referral directly; nurses could deliver ECT in the absence of a psychiatrist on site; nurses could run the service independently without a named psychiatrist for the clinic taking decisions about patient treatment and care. This gap in research literature needs to be addressed, especially at a time when some ECT treatment centres have closed leading to a decrease in the availability and use of ECT [24].

Theme 2: During the COVID-19 pandemic, nurses report an increased sense of teamwork among staff, with team members helping one another.

Nurses form the largest component of the hospital workforce and have played a vital role in caring for patients during the pandemic. Schroeder [4] described an increased sense of teamwork among staff, staff helping one another and in doing so felt that "we are all in this together". The need to adjust to an ever fluid 
challenging situation during COVID-19, the team at Berrywood found they had to adapt to the pandemic. On reflection, both nurses felt twice weekly huddles and debriefings at the beginning and the end of each shift boosted morale and the mental health wellbeing among team members, bringing a clear sense of cohesion and support. The authors have considered that, within their Trust, staff well-being was promoted as a priority during the pandemic and managers were encouraged to allow time and resources to provide this.

Schroeder et al. [25] and Sienaert et al. [4] found that nurses may accept their role during a pandemic as their duty; however, many of them have taken on roles previously performed by other colleagues, in this case the doctors administering ECT. Both nurses felt a pride in stepping up to the challenge. They felt their efforts had been recognized and that their confidence strengthened in the process. The Kings Fund [26] highlighted the need for belonging, to feel valued, respected and supported. This pride and sense of belonging requires an organisational commitment that identifies the need for staff protection especially when, in this case, they take on roles historically performed by doctors. Kontoangelos et al. [27] suggests healthcare staff may experience moral injuries of which they may become reluctant to speak about openly. Future research on lessons learnt during the pandemic will inform future emergency provision in healthcare, the need for supporting structures and processes should not just offer psychological and emotional support for those that seek out resources but that they capitalise on collaboration and teamwork to identify those nurses that do not recognise or prioritise their own need for support during the pandemic [25] [27]. It is likely due to the pandemic we may experience a future shortage of health professionals due to burnout and mental exhaustion [28].

Theme 3: The nurse's ability to manage psychologically trying situations and ethical dilemmas are dependent on education, individual knowledge, and experience.

Experienced ECT nurses are shown to be able to manage difficult situations, they are not only dependent on their education but also how well an organisation functions and the competence and professionalism of their colleagues [15]. Both nurses were supported by their teams and their NHS TRUST to advance their education enabling them to manage complex situations which in turn has led to enhanced patient care.

Both reflectors are experienced senior nurses with advanced abilities to reflect on their practice on a professional and personal level. Ericsson et al. [29] suggests greater emphasis should be placed upon performance in the real-life clinical setting rather than upon advanced education, suggesting nurses' self-assessment of their own professional abilities show reliable increases with age and professional experience. The OECD [16] states the United Kingdom has placed a greater importance on designing specific training programmes for nurses, wishing to expand their practice in certain areas by relying more on relevant work experience to promote nurses to more advanced positions. The OECD [16] suggests that healthcare must invest in preparing both nurses and doctors for future close 
collaboration and teamwork to enhance the more cost-effective advance nursing roles. This theme has been re-iterated by the Kings Fund [26] stating that nurses need to feel they have autonomy to be able to act consistently with their values, feeling they have real influence and voice across their organisations and within their teams. The Kings Fund [26] suggests hospital leaders should ensure the retention of experienced nursing staff through career pathways and role development, thus passing on their experience to support those early on in their career.

\section{Limitations}

There was not distance and separation during the data analysis due to dual roles of being a participant's voice and the researcher; however, it is the reader that judges the trustworthiness of the account [30]. The authors seek to demonstrate accuracy by utilising their own rich experiences in the phenomenon studied and demonstrated the skills needed to describe events through reflection. The analysis seeks to establish to the reader the "red thread" running through the reflections, guiding readers through the descriptive themes and meanings [31]. Further candidates would have been more customary to enlist to the study however, few mental health staff work within ECT and this select type of training is even scarcer.

\section{Conclusions}

This study has shown that positive feedback from patients and colleagues has enhanced nurse's professional and personal development. The nurses placed this feedback above any medical and technical skills they had acquired. Both nurses displayed a close professional relationship with their patients, valuing their thoughts and expressed feelings. This combined with effective team collaboration encouraged the nurses to progress in their practice with confidence. This is supported by research that suggests advanced nurse practice produces a high patient satisfaction rate, mainly due to the nurse devoting more time with each patient [6] [19] [20]. Both reflectors are experienced senior nurses. They were encouraged by management and their colleagues to advance their education empowering them to be able to manage more complex clinical decision-making. This theme has been reinforced by the Kings Fund [26] stating that nurses need to feel they have autonomy to be able to act consistently with their values, feeling they have real influence and voice across their organisations and within their teams. How a health organisation functions can influence their staff's professionalism and competence? There is a lot of literature and policy on advanced practice nursing roles that lacks real definition which, if supported by the NHS, would strengthen the identity of nurses that deliver autonomous evidenced-based high quality care.

COVID-19 pandemic poses a public health emergency unparalleled to anything in recent history [25]. This study demonstrated an increased sense of teamwork among nursing staff inspiring a "we are all in this together" type of 
approach. Both nurses showed pride in stepping up to the challenges posed, by replacing the junior doctors and releasing them for other duties. They felt their efforts had been recognised by their peers and their confidence strengthened. Research has suggested that nurses identified as experiencing the pressures of COVID-19 need psychological and emotional support through capitalising on collaboration and teamwork. However, NHS trusts also need to identify those nurses that do not recognise or prioritise their own needs for support during the pandemic.

Reflection and self-evaluation are shown to enhance personal as well as professional development [15]. This study shows the impact of the patients' voice on the nurses' confidence to progress forward with pride; perhaps future focus should be considered on integrating the patient experience to heighten team moral during difficult times. The importance of supporting structures and processes related to nursing care delivery during public health emergencies need to be recognised and developed to support nurses taking on new advanced roles and, in the process, enhancing their expertise and competence, which in turn must benefit patient care. It is time to utilise and expand advanced nursing practice within ECT treatment.

\section{Acknowledgements}

We thank Dr Jani, Dr Sabih, Morgan Bland (student nurse) and Chris Griffiths for their help and support

\section{Conflicts of Interest}

The authors declare no conflicts of interest regarding the publication of this paper.

\section{References}

[1] Bryson, E.O. and Aloysi, A.S. (2020) A Strategy for Management of Electroconvulsive Therapy Patients during the COVID 19 Pandemic. The Journal of ECT, 36, 149-151. https://doi.org/10.1097/YCT.0000000000000702

[2] Espinoza, R.R., Kellner, C.H. and McCall, W.V. (2020) Electroconvulsive Therapy during COVID-19: An Essential Medical Procedure-Maintaining Service Viability and Accessibility. The Journal of ECT, 36, 78-79.

https://doi.org/10.1097/YCT.0000000000000689

[3] Gil-Badenes, J., Valero, R., Valentí, M., Macau, E., Bertran, J.M., Claver, G., Bioque, M., Baeza, I., Bastidas Salvado, M., Mencia, M.L., Pacchiarotti, I., Bernardo, M. and Vieta, E. (2020) Electroconvulsive Therapy Protocol Adaptation during the COVID-19 Pandemic. Journal of Affective Disorders, 276, 241-248.

https://doi.org/10.1016/j.jad.2020.06.051

[4] Sienaert, P., Lambrichts, S., Popleu, L., Van Gerven., E., Buggenhout., S. and Bouckaert, F. (2020) Electroconvulsive Therapy During COVID-19-Times: Our Patients Cannot Wait. The American Journal of Geriatric Psychiatry, 28, 772-775. https://doi.org/10.1016/j.jagp.2020.04.013

[5] Tor, P.C., Phu, A.H.H., Koh, D.S.H. and Mok, Y.M. (2020) Electroconvulsive 
Therapy in a Time of COVID-19. The Journal of ECT, 36, 80-85. https://doi.org/10.1097/YCT.0000000000000690

[6] Feldman, S., Bachman, J., Cuffel, B., Friesen, B. and McCabe, J. (2003) Advanced Practice Psychiatric Nurses as a Treatment Resource: Survey and Analysis. Administration and Policy in Mental Health, 30, 479-494. https://doi.org/10.1023/A:1025025600989

[7] Hill, B. (2017) Exploring the Development and Identity of Advanced Practice Nursing in the U.K. Nursing Management, 24, 36-40. https://doi.org/10.7748/nm.2017.e1607

[8] Rosedale, M. and Knight, C. (2015) The Role of the Psychiatric Mental Health Advanced Practice Registered Nurse in the Scope of Psychiatric Practice. The Journal of ECT, 31, 205-206. https://doi.org/10.1097/YCT.0000000000000250

[9] Hardy, S., Cornish, J., Leyden, J., Vaughan, J. J. and O'Neill-Kerr, A. (2015) Should Nurses Administer Electroconvulsive Therapy? The Journal of ECT, 31, 207-208. https://doi.org/10.1097/YCT.0000000000000272

[10] Nmc.org.uk (2021) https://www.nmc.org.uk/globalassets/sitedocuments/other-publications/benefits-ofbecoming-a-reflective-practitioner---joint-statement-2019.pdf

[11] Flanigan, C. (1954) The Critical Incident Technique. Psychological Bulletin, 4, 327-358.

[12] Graneheim, U.H. and Lundman, B. (2004) Qualitative Content Analysis in Nursing Research: Concepts, Procedures and Measures to Achieve Trustworthiness. Nurse Education Today, 24, 105-112. https://doi.org/10.1016/j.nedt.2003.10.001

[13] Krippendorff, K. (2013) Content Analysis: An Introduction to Its Methodology. Sage, London.

[14] Morse, J. (2008) Confusing Categories and Themes. Qualitative Health Research, 18, 727-728. https://doi.org/10.1177/1049732308314930

[15] Wihlborg, J., Edgren, G., Johansson, A. and Sivberg, B. (2017) Reflective and Collaborative Skills Enhances Ambulance Nurses' Competence-A Study Based on Qualitative Analysis of Professional Experiences. International Emergency Nursing, 32, 20-27. https://doi.org/10.1016/j.ienj.2016.06.002

[16] Delamaire, M. and Lafortune, G. (2010) Nurses in Advanced Roles: A Description and Evaluation of Experiences in 12 Developed Countries (OECD Health Working Papers No. 54). OECD Publishing, Paris.

[17] Sjölin, H., Lindström, V., Hult, H., Ringsted, C. and Kurland, L. (2015) What an Ambulance Nurse Needs to Know: A Content Analysis of Curricula in the Specialist Nursing Programme in Prehospital Emergency Care. International Emergency Nursing, 23, 127-132. https://doi.org/10.1016/j.ienj.2014.09.002

[18] Piil, K., Kolbaek, R. and Ottmann, G. (2012) The Impact of Expanded Nursing Practice on Professional Identity in Denmark. Clinical Nurse Specialist, 26, 329-335. https://doi.org/10.1097/NUR.0b013e31826e3f43

[19] EROS Project (1999) Training Nurse Practitioners for General Practice. The EROS Project Team. British Journal of General Practice, 49, 531-535.

[20] Horrocks, S. (2002) Systematic Review of Whether Nurse Practitioners Working in Primary Care Can Provide Equivalent Care to Doctors. BMJ, 324, 819-823. https://doi.org/10.1136/bmj.324.7341.819

[21] Kinnersley, P. (2000) Randomised Controlled Trial of Nurse Practitioner versus General Practitioner Care for Patients Requesting "Same Day" Consultations in 
Primary Care. BMJ, 320, 1043-1048. https://doi.org/10.1136/bmj.320.7241.1043

[22] Lane, L. and Minns, S. (2010) Empowering Advanced Practitioners to Set up Nurse Led Clinics for Improved Outpatient Care. Nursing Times, 106, 14-15.

[23] (2006) Modernising Nursing Careers. British Journal of Community Nursing, 11, 530-531. https://doi.org/10.12968/bjen.2006.11.12.22423

[24] McAllister, M., Crabb, J., Brodie, D. and Krishnadas, R. (2011) Effect of Closing Facilities on Electroconvulsive Therapy Use in Glasgow. The Journal of ECT, 27, 131-133. https://doi.org/10.1097/YCT.0b013e3181f665cd

[25] Schroeder, K., Norful, A., Travers, J. and Aliyu, S. (2020) Nursing Perspectives on Care Delivery during the Early Stages of the Covid-19 Pandemic: A Qualitative Study. International Journal of Nursing Studies Advances, 2, Article ID: 100006. https://doi.org/10.1016/j.ijnsa.2020.100006

[26] West, M. Bailey, S. and Williams, E. (2020) The Courage of Compassion Supporting Nurses and Midwives to Deliver High-Quality Care. Kings Fund, London.

[27] Kontoangelos, K., Economou, M. and Papageorgiou, C. (2020) Mental Health Effects of COVID-19 Pandemia: A Review of Clinical and Psychological Traits. Psychiatry Investigation, 17, 491-505. https://doi.org/10.30773/pi.2020.0161

[28] Florillo, A. and Gorwood, P. (2020) The Consequences of the COVID-19 Pandemic on Mental Health and Implications for Clinical Practice. European Psychiatry, 63, e32. https://doi.org/10.1192/j.eurpsy.2020.35

[29] Ericsson, K.A., Whyte, J. and Ward, P. (2007) Expert Performance in Nursing Reviewing Research on Expertise in Nursing within the Framework of the ExpertPerformance Approach. Advances in Nursing Science, 30, E58-E71.

https://doi.org/10.1097/00012272-200701000-00014

[30] Rolfe, G. (2006) Methodological Issues in Nursing Research, Validity, Trustworthiness and Rigour: Quality and the Idea of Qualitative Research. Journal of Advanced Nursing, 53, 304-310. https://doi.org/10.1111/j.1365-2648.2006.03727.x

[31] Graneheim, U.H., Lindgren, B.M. and Lundman, B. (2017) Methodological Challenges in Qualitative Content Analysis: A Discussion Paper. Nurse Education Today, 56, 29-34. https://doi.org/10.1016/j.nedt.2017.06.002 